Quim. Nova, Vol. 29, No. 5, 916-921, 2006

\title{
INFLUÊNCIA DA ESPESSURA NAS PROPRIEDADES DE ABSORÇÃO E EMISSÃO E NA MORFOLOGIA DE FILMES AUTOMONTADOS DE POLI(P-FENILENO VINILENO)
}

\author{
Erick Piovesan*, Ángel Alberto Hidalgo e Alexandre Marletta \\ Instituto de Física, Universidade Federal de Uberlândia, CP 593, 38400-902 Uberlândia - MG \\ Maria Leticia Vega \\ Instituto de Física de São Carlos, Universidade de São Paulo, CP 369, 13560-970 São Carlos - SP \\ Reinaldo Ruggiero \\ Instituto de Química, Universidade Federal de Uberlândia, CP 593, 38400-902 Uberlândia - MG
}

Recebido em 12/4/05; aceito em 8/11/05; publicado na web em 5/5/06

\begin{abstract}
INFLUENCE OF THE THICKNESS ON ABSORBANCE AND EMISSION SPECTRA AND SURFACE MORPHOLOGY OF SELFASSEMBLED POLY( $p$-PHENYLENE VINYLENE) FILMS. In this report, we studied the thickness effect on the optical and morphological properties of self-assembled (SA) poly(p-phenylenevinylene) (PPV) films, wich were processed with 5 and 75 layers from a PPV precursor polymer and dodecylbenzenesulfonate, and then, thermally converted at $230{ }^{\circ} \mathrm{C}$. The increase of the film thickness yielded more intense peaks in the vibrational spectral range. The electron-phonon coupling was quantified by the Huang - Rhys factor, that shows the effects on the polymer chain mobility in the interface substrate/polymer. A strong emission anisotropy $r=0.57$ was observed for the film with 5 layers of thickness decreasing to 0.34 for the film with 75 layers. Finally, the surface topology of the films was measured using Atomic Force Microscopy.
\end{abstract}

Keywords: poly( $p$-phenylene vinylene); self-assembled films; thickness effect.

\section{INTRODUÇÃO}

Os polímeros eletroluminescentes formam uma nova classe de materiais, denominados semicondutores orgânicos, com grande potencial de aplicação tecnológica, principalmente, na indústria de diodos emissores de luz (ou LEDs - "Light Emitting Diodes") 1,2 . Uma grande revolução no estudo desses materiais ocorreu em 1977 quando acidentalmente, no laboratório do Prof. H. Shirakawa, foram produzidos filmes de poliacetileno que apresentavam um brilho metálico e características bem diferentes das até então conhecidas ${ }^{3}$. Atualmente, diversos polímeros têm sido sintetizados e estudados, apresentando um comportamento semicondutor/condutor similar ao observado no poliacetileno. $\mathrm{O}$ maior avanço ocorreu depois do trabalho de R. Friend e colaboradores ${ }^{4}$, em 1990, que mostraram a possibilidade da fabricação de LEDs poliméricos tendo como camada ativa o poli( $p$-fenilenovinileno) (PPV). Hoje, depois de uma década de pesquisa, LEDs de PPV e seus derivados, denominados de PLEDs ("Polymer Light Emitting Diodes"), são fabricados em laboratórios mostrando elevada eficiência, longo tempo de vida e, o mais importante, emissão em cores variadas ${ }^{5,6}$.

A procura por novos materiais poliméricos tem como primeiro estágio a escolha da rota de síntese química, e a possibilidade de obtenção de filmes finos é de fundamental importância para um estudo sistemático das suas propriedades ópticas e eletrônicas. Em particular, o PPV, por não possuir grupos químicos laterais, apresenta uma baixa solubilidade em solventes orgânicos, o que dificulta seu processamento em solução. Recentemente, em 1987, foi possível desenvolver uma rota de síntese química do PPV denominada de "rota do precursor solúvel" , na qual o poli(cloreto de tetraidrotiofeno de xililideno) (PTHT) era o precursor do PPV. Após o processamento do PTHT em forma de filme, este foi submetido a

*e-mail: piovesan@fafis.ufu.br uma etapa de eliminação térmica, a uma temperatura de $300{ }^{\circ} \mathrm{C}$ por $6 \mathrm{~h}$, tal como descrito por Bradley ${ }^{7}$. Durante este processo, ocorre a eliminação do grupo lateral tetraidrotiofeno do PTHT, liberando o $\mathrm{Cl}^{-}$e, principalmente acima de $150{ }^{\circ} \mathrm{C}$, com um aumento substancial da termo-oxidação do PTHT. Estes efeitos atuam fortemente na construção de dispositivos opto - eletrônicos, como "LED’s", por ex., prejudicando a injeção de portadores e diminuindo o grau de conjugação efetivo ao longo da cadeia polimérica $^{8,9}$.

Os filmes poliméricos podem ser preparados por diversas técnicas, porém as mais utilizadas são as de espalhamento ("casting") e espalhamento rotativo ("spin-coating"). A desvantagem dessas técnicas é que não permitem um controle da ordem ou do alinhamento molecular final dos filmes, produzindo em geral filmes altamente desordenados. Neste sentido, diversas técnicas vêm sendo usadas para se obter um ordenamento molecular de materiais eletroluminescentes orgânicos, tais como estiramento e deposição por "Langmuir-Blodgett" (LB) $)^{10-15}$. Um caminho alternativo para a obtenção de filmes ultrafinos de PPV, com controle na deposição de monocamadas, é a técnica de automontagem (ou "self-assembly", $\mathrm{SA})^{16}$. A grande vantagem desta técnica quando comparada, por ex., com a técnica de Langmuir-Blodgett, é seu baixo custo de produção e fácil implementação, o que permite controle da espessura de cada camada molecular adsorvida no substrato, tal como nos filmes LB. Por outro lado, nos filmes SA, podem ser montadas facilmente camadas com diferentes materiais poliméricos, permitindo a formação de heteroestruturas ${ }^{17}$.

A topologia da superfície dos filmes poliméricos é um parâmetro importante a ser controlado no momento de se montar um dispositivo eletroluminescente (PLED). A qualidade dos contatos elétricos depende essencialmente da uniformidade da superfície da camada ativa, neste caso o polímero ${ }^{18}$. As características da distribuição de alturas na superfície dos filmes poliméricos dependem de vários fatores, tais como foi feita a síntese do polímero, das inte- 
rações polímero/substrato, da técnica de deposição do filme no substrato, entre outros. A análise na escala nanométrica, via Microscopia de Força Atômica (AFM) destas superfícies é uma ferramenta fundamental para se correlacionar as propriedades optoeletrônicas e morfológicas de filmes finos poliméricos ${ }^{19}$. Guo e colaboradore ${ }^{20}$ observaram que "LEDs" obtidos a partir de superfícies mais rugosas exigiam maior corrente de injeção de cargas para sua ativação. Isto mostra a importância de se analisar a morfologia de superfície dos filmes poliméricos para se construir dispositivos opto-eletrônicos com mais alta eficiência.

Neste trabalho, apresentamos um estudo do efeito da espessura e da interface substrato/polímero (neste caso, vidro/polímero) na forma de linha de absorção e de emissão dos filmes de PPV. Os filmes foram preparados utilizando-se uma rota de síntese alternativa que nos possibilitou a deposição de filmes automontados pelo uso do polímero precursor (PTHT) em conjunto com o sal de sódio do ácido dodecilbenzenosulfônico (DBS). Os filmes foram caracterizados por espectroscopia de absorção e de emissão polarizada. Efeitos de ordenamento molecular foram observados e quantificados calculando-se a anisotropia dos filmes. Uma análise do acoplamento elétron-fônons foi realizada calculando-se o parâmetro Huang-Rhys, que permitiu também caracterizar de forma mais completa a interação do PPV com o substrato. As imagens de AFM foram usadas para se estudar a influência do aumento da espessura do filme de PPV na morfologia da superfície. Este estudo foi realizado pela análise quantitativa das imagens utilizando como parâmetros a função distribuição de alturas, $\mathrm{p}(\mathrm{h})$, e a rugosidade média quadrática, $\sigma_{\text {rms }}$. Finalmente, com os dados obtidos, foi possível estabelecer uma correlação entre as propriedades de luminescência e os efeitos da interface substrato/polímero na qualidade final dos filmes de PPV.

\section{PARTE EXPERIMENTAL}

Os substratos usados neste trabalho foram preparados de forma a se obter uma superfície o mais uniforme possível em sua textura e livre de impurezas, eliminando a possibilidade de artefatos. Os substratos usados foram lâminas de vidro BK7, especialmente apropriadas para estudos espectroscópicos, opacas para comprimentos de onda menores que $350 \mathrm{~nm}$. As lâminas foram previamente lavadas com detergente, enxaguadas com água ultrapura (Milli-Q) e submetidas a um processo de hidrofilização, para se obter de uma superfície rica em grupos $\mathrm{OH}^{-}$, o que imprime a estas lâminas uma característica altamente polar. Este método consiste em duas etapas: mergulhar os substratos em uma solução de $\mathrm{H}_{2} \mathrm{SO}_{4}$ e $\mathrm{H}_{2} \mathrm{O}_{2}$ em uma razão $7: 3$ (v/v) por $30 \mathrm{~min}$ a $80{ }^{\circ} \mathrm{C}$ (conhecida como solução "piranha") e, em seguida, os substratos foram mergulhados em uma solução de água ultrapura, $\mathrm{H}_{2} \mathrm{O}_{2}$ e $\mathrm{NH}_{4} \mathrm{OH}$ a 0,1 molar, na razão $5: 1: 1(\mathrm{v} / \mathrm{v} / \mathrm{v})$ por $30 \mathrm{~min}$ a $80^{\circ} \mathrm{C}$. Após cada procedimento, as lâminas foram novamente lavadas com água ultrapura.

Os filmes de PPV foram obtidos a partir da técnica de SA, que utiliza o princípio da adsorção do material inicialmente em solução sobre uma superfície sólida. O procedimento usado para se obter os filmes consiste em mergulhar o substrato nas soluções de um poliânion e de um policátion sucessivamente por um tempo $\mathrm{t}_{1} \mathrm{e}$ $\mathrm{t}_{2}$, respectivamente ${ }^{16}$. Obtendo-se no final de cada ciclo, uma camada dupla (bicamada) que pode ser repetida até se atingir a espessura desejada após a otimização dos parâmetros físicos e químicos que envolvem esta técnica. Além de polieletrólitos, é possível também utilizar moléculas iônicas menores ${ }^{17,21}$, tais como o sal de sódio do ácido dodecilbenzenosulfônico (DBS - Figura 1) como poliânion ${ }^{22}$. Neste caso particular não temos a formação específica de uma bicamada, devido ao fato de que o DBS é uma molécula
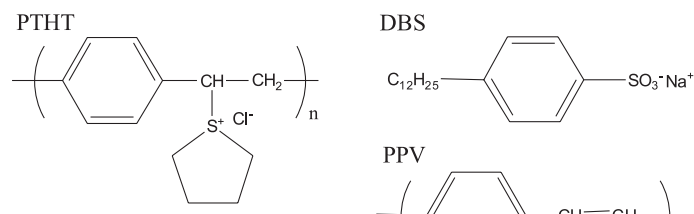

PPV

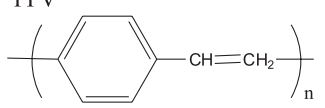

Figura 1. Estrutura química do polímero precursor poli(cloreto de tetraidrotiofeno de xililideno) (PTHT), do dodecilbenzenosulfônico (DBS) e do poli(p-fenileno vinileno). (PPV) após a etapa de termo eliminação

pequena que, além de realizar a troca iônica com o contra-íon $\mathrm{Cl}$, se difunde pela camada do polímero precursor ${ }^{17}$.

Neste trabalho, foi usado o DBS como ânion e o polímero precursor do PPV, poli(sulfonato de xilideno tetraidrotiofeno) (PTHT), como policátion, representados na Figura 1. O PTHT foi obtido segundo a rota de síntese química descrita por Bradley ${ }^{7}$. No caso particular do PTHT, pode-se obter filmes espessos por deposição não auto-limitada através desta técnica ${ }^{16}$. A deposição das camadas de PTHT/DBS foi feita utilizando-se soluções com concentrações iguais a $0,7 \mathrm{mg} \mathrm{mL}^{-1}$ e $10^{-1} \mathrm{M}$ para PTHT e DBS, respectivamente. Os tempos de imersão foram iguais a 1 min em cada solução, isto é, uma vez depositada a primeira camada de PTHT, o substrato seco foi mergulhado na solução de DBS por igual tempo, contabilizando desta forma uma camada depositada completa de PTHT/DBS, como ilustrado na Figura 2a. Nesta figura é apresentada uma representação esquemática da formação das multicamadas de PTHT/DBS. Depois de secar o substrato em fluxo suave de nitrogênio, novamente o ciclo é reiniciado, mergulhando-se o substrato com a camada previamente depositada na solução de PTHT e depois na de DBS, até completar o número de camadas desejado. A Figura $2 b$ ilustra o processo de deposição nas soluções poliiônicas via técnica de automontagem, observando a direção de mergulho, que será utilizada como referência neste trabalho. Foram obtidos filmes automontados PTHT/DBS com 5 e 75 camadas, os quais foram submetidos a tratamento térmico à $230{ }^{\circ} \mathrm{C}$ sob vácuo, para se obterem os filmes automontados de PPV (SA-PPV). Estes filmes foram utilizados para estudar a influência do número de camadas de PPV nas propriedades ópticas e na topografia da superfície.

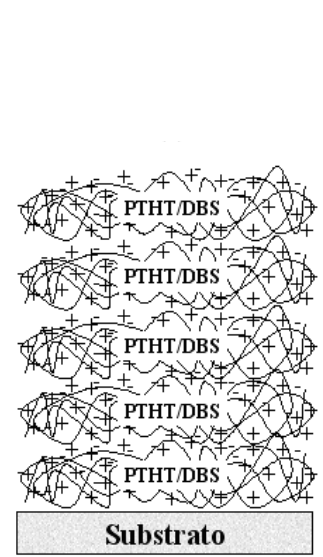

(a)

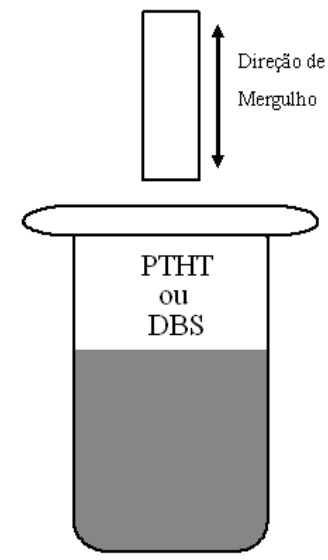

(b)
Figura 2. (a) Esquema de deposição de filmes via técnica de automontagem e (b) formação das camadas de PTHT/DBS

As propriedades de absorção na região do UV-VIS dos filmes foram obtidas utilizando-se um espectrômetro Shimatzu modelo UV-160. As medidas de emissão para as diferentes condições de 
polarização foram feitas na região espectral de 470 até $700 \mathrm{~nm}$, utilizando-se um espectrofluorímetro Hitachi F-4500 com excitação em $450 \mathrm{~nm}$, cuja polarização era controlada por polarizadores que permitiam a passagem de luz com polarização paralela (//) ou perpendicular $(\perp)$ à direção de mergulho (Figura $2 b$ ). Para obtenção dos espectros de fotoluminescência, os filmes foram posicionados de tal forma que o feixe de excitação incidia sobre a amostra a $45^{\circ}$; com este procedimento, evitou-se ao mesmo tempo a reflexão direta na fotomultiplicadora (o que pode ocasionar saturação da mesma) e a possibilidade de aberrações nos espectros, decorrentes da detecção de luz espalhada na superfície dos filmes.

$\mathrm{O}$ estudo da topografia da superfície foi realizado usando a técnica de Microscopia de Força Atômica (AFM). As imagens de AFM foram obtidas usando um microscópio de força atômica da Nanoscope $^{\circledR}$ IIIa Multimode ${ }^{\mathrm{TM}}$ no modo "tapping", utilizando uma ponta de prova de Si com uma constante de mola de 20-100 N/m e uma frequiência de oscilação entre 250 e $300 \mathrm{KHz}$. A área de varredura foi de $10 \times 10 \mu \mathrm{m}^{2}$ com taxa de $0,7 \mathrm{~Hz}$. A espessura dos filmes foi obtida por AFM seguindo o método descrito por Lobo e colaboradore $^{23}$ e consiste em riscar o filme no mesmo lugar até atingir o substrato e, assim, determinar a diferença de altura.

\section{RESULTADOS E DISCUSSÃO}

Na Figura 3 são mostradas as curvas de absorbância polarizada dos filmes de PPV/DBS com 5 e 75 camadas na região espectral entre 350 e $750 \mathrm{~nm}$. A direção paralela $\left(\mathrm{A}_{/ \mid}\right)$e perpendicular $\left(\mathrm{A}_{\perp}\right)$ das medidas referem-se ao estado de polarização da luz transmitida através dos filmes, tendo como referência a direção de mergulho dos filmes nos recipientes contendo PTHT e DBS (Figura 2b). A matriz polimérica pode ser descrita como um conjunto de cadeias com diferentes comprimentos de conjugação e a distribuição de conjugações pode ser descrita por uma gaussiana com um número médio $n$ de meros. Efetivamente, uma distribuição de segmentos conjugados mais larga ocasiona um alargamento das bandas do espectro de absorção e um deslocamento do máximo para o azul. O tratamento térmico durante a conversão do PTHT em PPV leva a um deslocamento do máximo da banda do espectro de absorção para o vermelho e um estreitamento de sua largura, com as cadeias de maior comprimento de conjugação localizadas no canto da banda de absorção, em torno de $510 \mathrm{~nm}$ (Figura 3). Desta forma, comparando-se os espectros de absorção obtidos com os filmes de 5 e

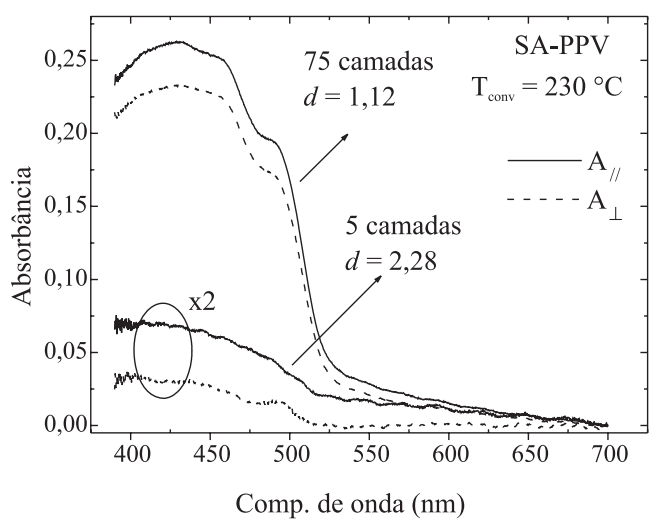

Figura 3. Absorção polarizada dos filmes SA PPV/DBS com 5 e 75 camadas. As direções $A_{/ /} e A_{\perp}$ referem-se, respectivamente, às direções paralela $e$ perpendicular à direção de mergulho dos filmes. Para melhor visualização, a intensidade de absorção dos filmes com 5 camadas foram multiplicadas pelo fator 2 (x2)
75 camadas, pode-se afirmar que o filme de 5 camadas apresenta uma distribuição de segmentos conjugados mais larga, com o centro da distribuição em comprimentos de conjugação menores. Porém, renormalizando todos os espectros para apresentarem a mesma intensidade em $440 \mathrm{~nm}$, observamos que não existe um deslocamento do canto da banda de absorção, o que é um indicativo de que apenas a largura e o número médio da distribuição de segmentos conjugados são modificados, deslocando o máximo do espectro de absorção para o azul.

A existência de um ordenamento no filme de PPV é manifestada pela diferença de intensidade das bandas de absorção em diferentes condições de polarização. A intensidade de absorção é maior quando o espectro é obtido com luz polarizada na direção de mergulho do substrato (Figura 3). A razão dicróica $\left(\boldsymbol{d}=\mathrm{A}_{/ /} / \mathrm{A}_{\perp}\right)$ calculada em $440 \mathrm{~nm}$ diminui de 2,28 para 1,12 quando a espessura do filme aumenta de 5 para 75 camadas, como pode ser visto na Tabela 2. Comparando com filmes altamente organizados, particularmente os obtidos pela técnica Langmuir-Blodgett, ${ }^{24}$ os filmes SA-PPV com 75 camadas apresentam uma razão dicróica 3,2 vezes menor. Este dado mostra que estes filmes são comparativamente pouco ordenados confirmando a hipótese de que, com o aumento do número de camadas, há um crescimento desordenado (ou randômico) do filme, propriedade intrínseca da técnica de automontagem. Esta desordem deve-se ao próprio mecanismo de formação dos filmes, onde a adsorção física dos polieletrólitos ocorre predominantemente por meio de interações do tipo dipolo-dipolo elétrico, van der Walls e pela formação aleatória de aglomerados moleculares distribuídos ao longo do plano do filme ${ }^{25}$. Assim, os efeitos iniciais da interface com o substrato são atenuados gradativamente com o aumento do número de camadas, sobressaindo os efeitos de volume. Porém, os filmes de poucas camadas apresentam um grau de ordenamento maior, induzido pelos efeitos da interface substrato/polímero.

Diferente das medidas de absorção óptica, as transições eletrônicas referentes aos fenômenos de emissão ocorrem apenas nos segmentos de maior conjugação, ou menor "Gap". Como este processo é seletivo (apenas as maiores cadeias participam da emissão), espera-se que sejam obtidas bandas mais estreitas e mais definidas. Assim, após a excitação óptica ou elétrica, o par elétronburaco (éxciton-polaron) migra para regiões com cadeias poliméricas de maior grau de conjugação via transferência de energia e/ou difusão de carga $^{26,27}$. Na Figura 4 são apresentados os espectros de fotoluminescência dos filmes SA-PPV com 5 camadas (Figura 4a) e 75 camadas (Figura 4b) em função da polarização da luz de excitação $\left(\mathrm{E}_{\mathrm{ex}}\right)$ e emissão $\left(\mathrm{E}_{\mathrm{em}}\right)$. Em geral, os espectros apresentam três bandas de emissão, correspondentes a uma transição denominada zero-fônon (altas energias) e duas réplicas de fônon (acoplamento elétron-fônon). Os modos vibracionais (ou fônons) do PPV, que se acoplam efetivamente com os elétrons $\pi$ são: o estiramento $\mathrm{C}=\mathrm{C}$ do grupo fenila $\left(1420,1517\right.$ e $\left.1600 \mathrm{~cm}^{-1}\right)$, o cisPPV $\left(868 \mathrm{~cm}^{-1}\right)$ e o trans-PPV $\left(962 \mathrm{~cm}^{-1}\right)^{28}$. Desta forma, observamos no espectro de emissão o desdobramento de uma banda principal em 510 nm nas réplicas em 545 e 590 nm (Figura 4); a diferença de energia entre cada uma destas bandas corresponde à energia vibracional relacionada aos números de onda dos modos vibracionais descritos acima. Com o aumento do número de camadas dos filmes de PPV/DBS, os espectros mostram que a posição central das bandas de emissão se desloca para o vermelho, de forma similar ao observado no espectro de absorção (Figura 3).

A intensidade relativa entre as bandas de emissão (réplicas de fônon e zero-fônon) pode ser relacionada ao acoplamento do estado excitado do polímero com os fônons da rede. Este acoplamento pode ser quantificado pelo parâmetro de Huang-Rhys $(S)^{3}$. A Ta- 

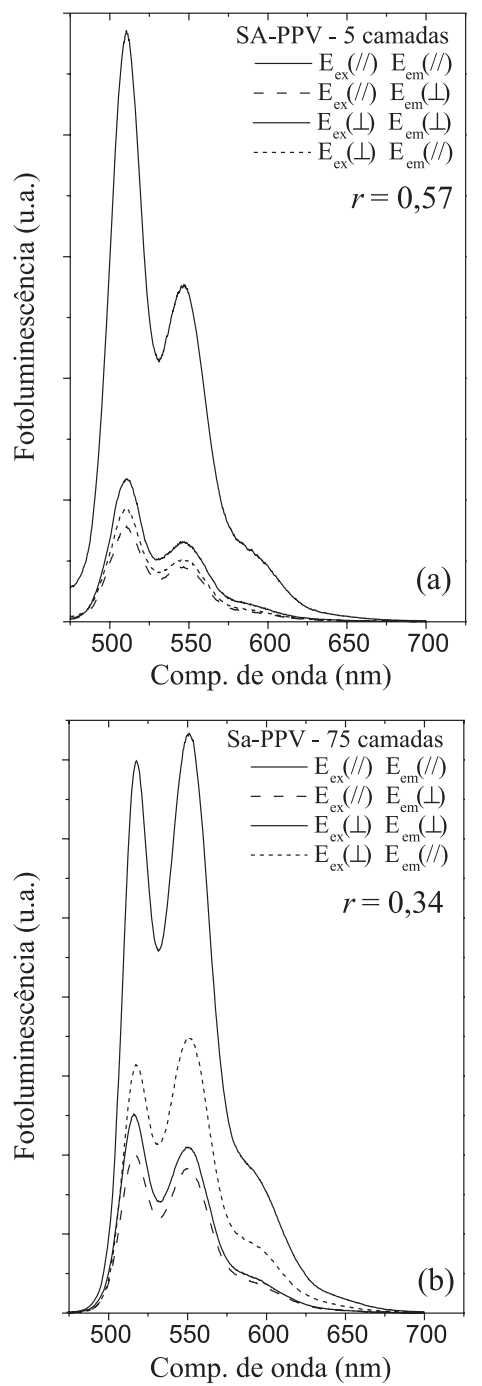

Figura 4. Espectros de fotoluminescência polarizada dos filmes SA de PPV/ DBS com 5 (a) e 75 (b) camadas

bela 1 mostra o valor de $\mathrm{S}$ em função da polarização de excitação $\left(\mathrm{E}_{\mathrm{ex}}\right)$ e emissão $\left(\mathrm{E}_{\mathrm{em}}\right)$. Nota-se um aumento significativo de $S$ com o aumento do número de camadas (ou espessura) do filme, permanecendo praticamente inalterado para as diferentes polarizações. Os valores de $S$ relativamente altos para os filmes de SA-PPV/DBS podem estar relacionados a três fatores: ao aumento do número de defeitos estruturais ao longo da cadeia polimérica; a uma maior interação entre cadeias e, a efeitos de volume. Como os filmes de SA-PPV/DBS com 5 e 75 camadas foram preparados sob as mesmas condições, espera-se que tenham sido produzidos concentrações de defeitos estruturais similares ao longo da cadeia do PPV. Assim podem-se descartar os dois primeiros itens supostos acima. Como existe uma diferença de espessura significativa entre os dois

Tabela 1. Valores de anisotropia calculados por integração dos espectros de emissão apresentados na Figura 4

\begin{tabular}{|c|c|c|}
\hline Polarização & SA-PPV5 camadas & SA-PPV 75 camadas \\
\hline $\mathrm{E}_{\mathrm{ex}}(/ /) \quad \mathrm{E}_{\mathrm{em}}(/ /)$ & $S \approx 0,6$ & $S \approx 1,1$ \\
\hline $\mathrm{E}_{\mathrm{ex}}(/ /) \quad \mathrm{E}_{\mathrm{em}}^{\mathrm{em}}(\perp)$ & $S \approx 0,6$ & $S \approx 0,9$ \\
\hline $\mathrm{E}_{\mathrm{ex}}(\perp) \quad \mathrm{E}_{\mathrm{em}}(\perp)$ & $S \approx 0,6$ & $S \approx 0,8$ \\
\hline $\mathrm{E}_{\mathrm{ex}}(\perp) \quad \mathrm{E}_{\mathrm{em}}(/ /)$ & $S \approx 0,5$ & $S \approx 1,1$ \\
\hline
\end{tabular}

filmes, o aumento de $\mathrm{S}$ pode estar relacionado a efeitos de volume e/ou de interface. Em outras palavras, a interação do PPV com o substrato limita seus graus de liberdade vibracionais, o que é equivalente a uma diminuição na temperatura, ocasionando uma diminuição de $\mathrm{S}$.

Observa-se também que $\mathrm{S}$ praticamente independe da configuração dos polarizadores, mostrando que a distribuição de tamanhos de segmentos conjugados em ambas direções (paralela e perpendicular) no plano do filme é supostamente a mesma. Como já discutido nos espectros de UV-VIS, existe uma anisotropia que deveria ser menor no caso do filme mais espesso, com 75 camadas. É interessante notar que este resultado não está em contradição com as observações feitas sobre os resultados do cálculo do parâmetro S, pois os espectros UV-VIS monitoram apenas o estado fundamental, e os espectros de emissão monitoram o estado excitado. Em outras palavras, na emissão estão envolvidos diferentes processos de transferência de energia, que fazem com que as transições eletrônicas aconteçam principalmente nas cadeias de maior comprimento de conjugação. A independência dos valores de $\mathrm{S}$ com a configuração dos polarizadores é um indicativo de que o número médio de conjugação dos segmentos, onde acontece a emissão, é o mesmo nas direções paralela e perpendicular do filme. Entretanto, cadeias poliméricas com segmentos conjugados maiores deveriam apresentar valores de $S$ menores $^{3}$. Assim, a variação de $\mathrm{S}$ e o deslocamento do máximo de absorção para os filmes SA-PPV/DBS com o aumento da espessura não apresentam contradição, pois os segmentos envolvidos nos processos de absorção e de emissão são distintos.

Outro parâmetro importante que caracteriza a distribuição de orientação dos dipolos de emissão é a anisotropia da luminescência, definida por $^{29}$ :

$\mathrm{r}=\frac{\mathrm{I}_{/ /, / /}-\mathrm{GI}_{/ /, \perp}}{\mathrm{I}_{/ /, / /}+2 \mathrm{GI}_{/ /, \perp}}$

onde $\mathrm{I}_{\mathrm{Ex}, \mathrm{Em}}$ é a intensidade de emissão e os sub-índices indicam as polarizações, paralela $(/ /)$ e perpendicular $(\perp)$ de excitação $\left(\mathrm{E}_{\mathrm{ex}}\right)$ e emissão $\left(\mathrm{E}_{\mathrm{em}}\right)$. $\mathrm{O}$ fator $\mathrm{G}=\mathrm{I}_{\perp, /} / \mathrm{I}_{\perp, \perp}$ é o parâmetro de correção instrumental, pois os diferentes componentes óticos presentes no espectrofluorímetro apresentam uma transmitância que depende da direção de polarização da luz. Assim, há necessidade de utilizar este parâmetro de correção para poder comparar as intensidades da emissão polarizada dos filmes SA-PPV/DBS.

A Equação 1 junto com a definição do parâmetro $G$ mostram claramente a necessidade de se obter quatro espectros (as quatro configurações possíveis dos polarizadores) e assim determinar o parâmetro de anisotropia. Note que $r$, Equação 1, permanece inalterada ante uma troca dos eixos, ou seja, // $\rightarrow \perp$ e $\perp \rightarrow$ // (equivalente a uma rotação de $90^{\circ}$ da amostra); em outras palavras, $r$ é um número que caracteriza o grau de ordenamento da amostra, porém, não indica qual é a direção do ordenamento na amostra. $\mathrm{O}$ indicativo da direção de orientação das cadeias poliméricas no substrato está contido nos espectros de absorção, que indicaram que a quantidade de cadeias poliméricas na direção de mergulho (paralelo) é maior que na direção perpendicular. Os valores encontrados de $r$ são 0,57 e 0,34 para os filmes de 5 e 75 camadas, respectivamente. A diminuição substancial deste parâmetro, que pode assumir valores entre 0 e 1 , em função do aumento do número de camadas do filme indica, claramente, a presença de uma correlação entre anisotropia do filme e as interações do PPV com o substrato. Ou seja, o filme mais espesso apresenta propriedades de "volume", onde os segmentos conjugados devem apresentar orien- 
tações mais isotrópicas, devido ao processo de deposição. Este dado está de pleno acordo com as características da técnica de deposição de filmes automontados que, em princípio, ocorre por deposição aleatória via aglomerados. O valor de 0,34 para $r$ é muito próximo do valor máximo esperado $(0,4)$ para uma amostra na qual os dipolos de transição são colineares e as moléculas não apresentam orientação preferencial ${ }^{29}$. Por outro lado, valores altos de anisotropia $(>0,4)$ foram também obtidos por Schwartz e colaboradores ${ }^{30}$ para filmes de MEH-PPV orientados por confinamento em um sistema poroso. Neste trabalho, os autores obtiveram a relaxação da anisotropia do sistema e observaram que com o tempo a anisotropia tende a aumentar, indicando que a energia migra para segmentos de maior conjugação e preferencialmente orientados na direção dos poros.

Na Figura 5 são apresentadas as imagens obtidas por AFM dos filmes de SA-PPV com 5 e 75 camadas. Na Tabela 2 são apresentados os valores da rugosidade média quadrática $\left(\sigma_{\mathrm{RMS}}\right)$ e a espessura ( $l$ ) destes filmes, onde se nota um aumento de aproximadamente 6,5 vezes no parâmetro $\sigma_{\text {RMS }}$ com o aumento do número de camadas. Dos dados de espessura, obteve-se uma espessura média por camada de PPV+DBS de 2,8 nm. Uma análise visual das imagens de AFM mostra que o filme de 5 camadas (Figura 5a) apresenta

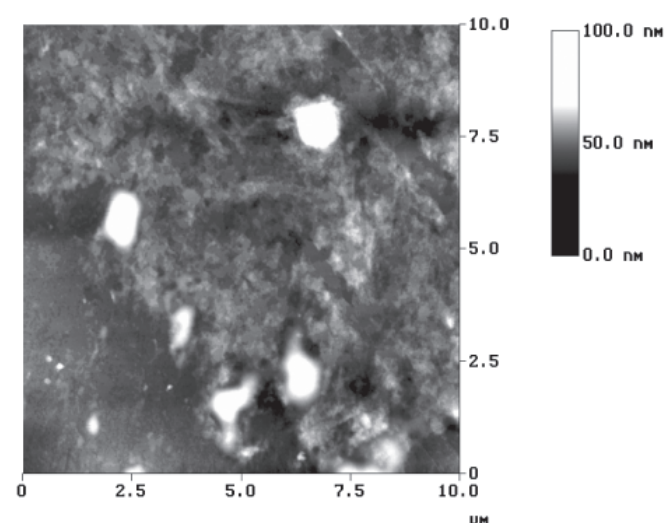

(a)

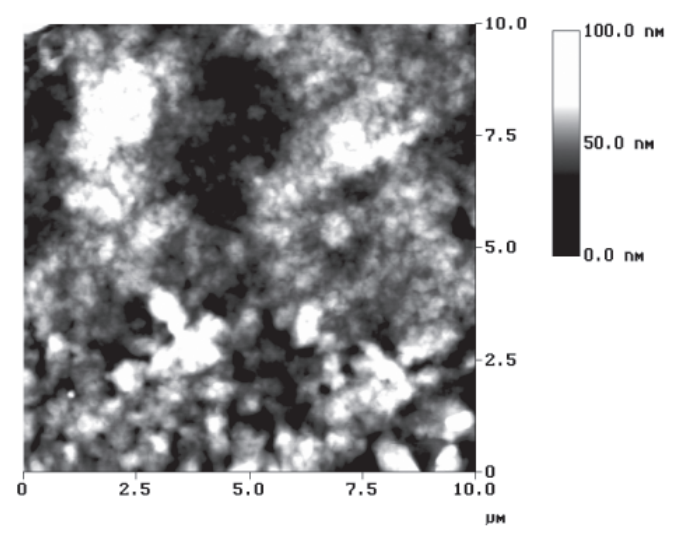

(b)

Figura 5. Imagens de AFM dos filmes SA de PPV/DBS com 5 (a) e 75 (b) camadas

Tabela 2. Valores da rugosidade média quadrática $\left(\sigma_{R M S}\right)$ e espessura (l) dos filmes SA de PPV/DBS de 5 e 75 camadas

\begin{tabular}{ccc}
\hline Camadas PPV/DBS & $\sigma_{R M S}(\mathrm{~nm})$ & $l(\mathrm{~nm})$ \\
\hline 5 & 6 & 14,5 \\
75 & 44 & 206,3 \\
\hline
\end{tabular}

regiões com diferentes texturas; já o de 75 camadas apresenta um número grande de aglomerados com alturas superiores a $50 \mathrm{~nm}$ (Figura 5b). Deste modo, pode-se afirmar que a morfologia dos filmes SA-PPV/DBS depende da espessura e é fortemente influenciada pelos efeitos de interface.

Outro estudo que pode ser realizado a partir das imagens de AFM é a análise da distribuição de alturas do perfil de superfície dos filmes. A função distribuição de alturas é construída a partir da medida das alturas em todos os pontos da imagem. A distribuição de alturas $\mathrm{P}(\mathrm{h})$ pode ser ajustada usando a seguinte equação:

$P(h)=\frac{A}{w^{*} \sqrt{2 * \pi}} \exp \left\{\frac{-\left(h-h_{c}\right)^{2}}{2 * w^{2}}\right\}$

onde $A$ é a área da distribuição, $w$ é a largura da distribuição e $h_{c}$ é o valor médio das alturas $h$. Na Figura 6 são mostradas as distribuições de altura dos filmes SA-PPV em função da freqüência relativa normalizada pelo número total de pontos e a largura do intervalo $\Delta h$. Esta normalização dos histogramas garante que a área abaixo da curva experimental seja igual a 1.

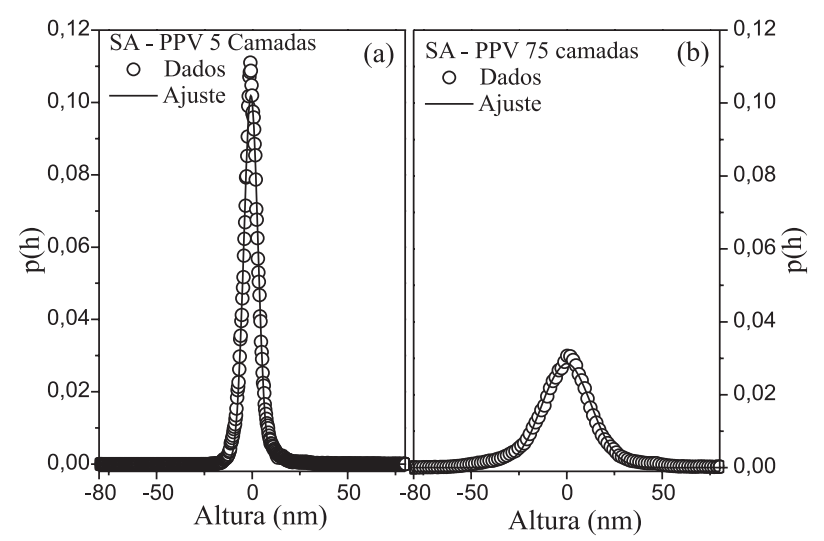

Figura 6. Gráfico (círculos abertos) da distribuição de alturas $P(h)$ (Equação 2) dos filmes SA de PPV/DBS de 5 (a) e 75 (b) camadas. A linha contínua refere-se ao ajuste dos dados utilizando a Equação 2

De acordo com a literatura ${ }^{32}$, para que uma superfície seja considerada "normal", a largura da distribuição de alturas $(w)$ deve ser igual à rugosidade média quadrática $\left(\sigma_{\mathrm{RMS}}\right)$, e a área da distribuição de alturas $(A)$ deve ser igual à área da curva experimental $\left(\mathrm{A}_{\text {exp }}\right)$. Na Tabela 3 são mostrados os valores obtidos do ajuste das distribuições de alturas (Figura 6) usando a Equação 2. Ao comparar a largura da distribuição $w$ (Tabela 3) com o valor da $\sigma_{\text {RMS }}$ (Tabela 2), observa-se que à medida que a espessura do filme (número de camadas) aumenta, os valores tornam-se diferentes do esperado para uma superfície "normal", indicando que a distribuição de alturas deixa de ser descrita por uma gaussiana. O fato do sistema não ser descrito por uma distribuição gaussiana indica que o crescimento das regiões de maior altura na superfície dos filmes deixa

Tabela 3. Parâmetros de ajuste da distribuição de alturas $\mathrm{P}(\mathrm{h})$ (Equação 2) dos filmes SA de PPV/DBS de 5 e 75 camadas

\begin{tabular}{cccc}
\hline Camadas & $h_{c}(\mathrm{~nm})$ & $w(\mathrm{~nm})$ & $\mathrm{A}$ \\
\hline PPV/DBS & & & \\
5 & $-0,635$ & 7,27 & 0,93 \\
75 & $-0,014$ & 25,4 & 0,91 \\
\hline
\end{tabular}


de ser completamente aleatória. Ou seja, existe uma correlação entre a localização dos "picos" e "vales" na superfície dos filmes SA-PPV/DBS. Isto fica mais claro quando se observa a Figura 5, onde é possível identificar o crescimento de "aglomerados" no filme mais espesso.

\section{CONCLUSÕES}

Em resumo, os espectros de absorção no UV-VIS dos filmes SA-PPV/DBS mostram claramente que existe uma direção preferencial de orientação das cadeias poliméricas, que é paralela à direção de mergulho do substrato no processo de deposição dos filmes. Por outro lado, a ausência de deslocamento do canto da banda de absorção em conjunto com os resultados do parâmetro de Huang - Rhys mostram que o processo de emissão acontece em cadeias poliméricas de comprimento de conjugação similares, independente da direção no plano do filme. Desta forma, a função distribuição que descreve os comprimentos de conjugação do polímero em cada direção é a mesma, porém, o número total de cadeias poliméricas em uma direção é diferente de outra, sendo maior na direção de mergulho do filme. Aumentando a espessura do filme, o parâmetro de Huang - Rhys aumenta, indicando um menor efeito da interface substrato/filme e concordando com o resultado do parâmetro de anisotropia $r$, que diminui com a espessura do filme.

$\mathrm{O}$ estudo das propriedades ópticas e morfológicas da superfície dos filmes de SA-PPV/DBS, em função da espessura mostrou que, apesar de se utilizar uma técnica simples como a de automontagem, é possível obter filmes com razoável ordenamento molecular. Além disso, este ordenamento depende da espessura da camada polimérica, com um alcance da ordem de centenas de ângstrons (para o filme com 75 camadas). Este dado é de fundamental importância quando se objetiva a produção de dispositivos eletroluminescentes mais eficientes. Neste ponto é importante observar que os PLEDs são construídos, geralmente, com filmes de espessura da ordem de $100 \AA$ A. Neste limite de espessura, pode-se afirmar que, no caso de filmes SA-PPV sob substrato de vidro, a camada ativa ainda está sobre influência da interface, o que limita o aumento da eficiência de emissão radiativa por moléculas livres de defeitos provocados não intencionalmente. Além disto, a análise das imagens de AFM mostrou que o aumento da espessura da amostra introduz um alargamento substancial na distribuição de alturas e, tal como descrito anteriormente, ela se afasta de uma distribuição gaussiana. De acordo com a literatura, uma distribuição de probabilidades descrita por uma gaussiana indica que os eventos não possuem correlação entre $\mathrm{si}^{33}$. A princípio, para um filme fino, esta distribuição se aproxima mais de uma distribuição gaussiana, o que indica que seu crescimento é completamente aleatório. Entretanto, quando a espessura aumenta, o processo de adsorção do PTHT apresenta uma correlação entre as alturas, provavelmente dependendo da posição da camada anterior adsorvida. Isto está refletido no fato do crescimento de "aglomerados", claramente visíveis na Figura $5 b$.

Os resultados mostrados neste artigo indicam que filmes muito espessos apresentam propriedades ópticas de volume, em outras palavras, com pouca influência da interface polímero/substrato, porém, o aumento da espessura da camada polimérica causa um crescimento desordenado das cadeias e com regiões de aglomerados na superfície. Este último fato é de grande importância na construção de PLEDs, pois superfícies com grandes variações de alturas (vales e picos) irão implicar em uma deposição ruim da camada metálica (eletrodo) utilizada como injetora de cargas em PLEDs.

\section{AGRADECIMENTOS}

Ao apoio do Prof. Dr. M. Tabak do Instituto de Química de São Carlos - USP por nos emprestar seu laboratório. Agradecemos também o apoio financeiro das agências brasileiras CAPES, CNPq, IMMP/MCT e FAPESP.

\section{REFERÊNCIAS}

1. van Hutten, P.F.; Krasnikov, V.V.; Hadziioannou, G.; Acc. Chem. Res. 1999, 32, 257.

2. http://w4.siemens.de/FuI/en/archiv/pof/index.html, acessada em Novembro 2004.

3. Yu, J.; Hsu, J. H.; Chuang, K. R.; Chao, C. L.; Chen, S. A.; Kao, F. J.; Fann, W. S.; Lin, S. H.; Synth. Met. 1995, 74, 7.

4. Burroughes, J. H.; Bradley, D. D. C.; Brown, A. R.; Marks, R. N.; Mackay, K.; Friend, R. H.; Burn P. L.; Holmes, A. B.; Nature 1990, 347, 539.

5. Forrest, S.R.; Burrows, P.E.; Shen, Z.; Gu, G.; Bulovic, V.; Thompson, M.E.; International Conference on Electroluminescence of Molecular Materials and Related Phenomena, Synth. Metals, Fukuoka, Japan, 1997.

6. Hosokawa, C.; Eida, M.; Matsuura, M.; Fukuoka, K.; Nakamura, H.; Kusumoto, T.; International Conference on Electroluminescence of Molecular Materials and Related Phenomena, Synth. Metals, Fukuoka, Japan, 1997.

7. Bradley, D. D. C.; J. Phys. D: Appl. Phys. 1987, 20, 1389.

8. Nguyen, T. P.; de Vos, S.; Vacuum 1996, 47, 1153.

9. Cumpston, B. H.; Jensen, K. F.; Trends Pol. Sci. 1996, 4, 151.

10. Hagler, T. W.; Pakbaz, K.; And Heeger, A. J.; Phys. Rev. B: Condens. Matter Mater. Phys. 1994, 49, 968.

11. Cimrová, V.; Remmers, M.; Neher D.; Wegner, G.; Adv. Mater. 1996, 8, 146.

12. Jung, G. Y.; Pearson, C.; Horsburg, L. E.; Samuel, I. D. W.; Monkman, A. P.; Petty, M. C.; J. Phys. D.: Appl. Phys 2000, 33, 1029.

13. Wu, Z.; Wu, S.; Liang, Y.; Langmuir 2001, 17, 7267.

14. Wu, Z. K.; Wu, S. X.; Liao, J. H.; Fu, D.G., Liang, Y.Q.; Synth. Met. 2002, $130,35$.

15. Ferreira, M.; Constantino, C. J. L.; Olivati, C. A.; Vega, M. L.; Balogh, D. T.; Aroca, R. F.; Faria, R. M.; Oliveira Jr., O. N.; Langmuir 2003, 19, 8835.

16. Oliveira Jr, O. N.; Zucolotto, V.; Balasubramanian, S.; Li, L.; Kumar, J.; Tripathy, S. K.; In: Tripathy, S. K.; Kumar, J.; Nalwa, H. S., Orgs.; Handbook Of Polyelectrolytes; New York, 2002, vol. 1, p. 1-37.

17. Marletta, A.; Piovesan, E.; de Souza, N. C.; Olivati, C.A.; Balogh, D. T.; Dantas, N. O.; Faria, R. M.; Oliveira Jr., O. N.; J. Appl. Phys. 2003, 94, 5592.

18. Surin, M.; Hennebicq, E.; Ego, C.; Marsitzky, D.; Grimsdale, A. C.; Müllen, K.; Brédas, J. L.; Lazzaroni, R.; Leclère, P.; Chemical of Materials 2004, 16,994

19. Assender, H.; Bliznyuk, V.; Porfyrakis, K. Science 2002, 297, 973.

20. Guo, T. F.; He, G.; Pyo, S.; Yang, Y.; App. Phys. Lett. 2002, 80, 4042.

21. Melo, R. M.; Marletta, A.; de Souza, N. C.; Oliveira Jr., O. N.; Faria, R. M.; Dantas, N. O.; Quim. Nova 2003, 26, 177.

22. Marletta, A.; Castro, F. A.; Borges, C. A. M.; Oliveira Jr., O. N.; Faria, R. M.; Guimarães, F. E. G. Macromolecules 2002, 35, 9105.

23. Lobo, R. F. M.; Pereira-da-Silva, M. A.; Raposo, M.; Faria, R. M.; Oliveira Jr., O. N.; Nanotechnology 1999, 10, 389.

24. Marletta, A.; Gonçalves, D.; Oliveira Jr, O.N.; Faria R.M.; Guimarães, F.E.G.; Macromolecules 2000, 33, 5886.

25. Ivov, T.; Decher, G.; Möhaward, H.; Langmuir 1993, 9, 481.

26. Greenham, N. C.; Friend, R. H.; Em Quantum Theory of Solids; Peierls, R. E., ed.; Oxford 1995, vol. 49, cap. 1.

27. Cazati, T.; Dissertação de Mestrado, Universidade de São Paulo, Brasil, 2003.

28. Rakvié, D.; Kostié, R.; Gribov, L. A.; Davidova, I. E.; Phys. Rev. B: Condens. Matter Mater. Phys. 1990, 41, 10744

29. Lakowicz, J. R., Principles of Fluorescence Spectroscopy, New York Plenum Press, 1983.

30. Schwartz, B. J.; Nguyen, T. P.; Wu, J.; Tolbert, S. H.; Synth. Met. 2001, $116,35$.

31. Schäfer, O.; Greiner, A.; Pommerehne, J.; Guss, W.; Vestweber, H.; Tak, H. Y.; Bässler, H.; Schmidt, C.; Lüssem, G.; Schartel, B.; Stümpften, V.; Wendorff, J. H.; Spiegel, S.; Möller, C.; Spiess, H. W.; Synth. Met. 1996, 82,1 .

32. Bennett, J. M.; Mattsson, L.; Introduction to surface roughness and scattering, Optical Society of America: Washington D.C., 1999.

33. Vuolo, J. H.; Fundamentos da Teoria de Erros, Edgard Blücher, 1992. 